Research Article

\title{
Application of Linear Gradient Solvent System in Centrifugal Partition Chromatography Facilitating Bioassay-Guided Fractionation of Yongdamsagan-Tang, Traditional Oriental Decoction
}

\author{
Ji Hoon Kim $\mathbb{D}^{1},{ }^{1}$ Eun Ju Jung, ${ }^{1}$ Yun Jung Lee, ${ }^{1}$ Chul Young Kim $\mathbb{D}^{1},{ }^{1}$ and Je-Seung Jeon $\mathbb{D}^{1,2}$ \\ ${ }^{1}$ College of Pharmacy and Institute of Pharmaceutical Science and Technology, Hanyang University, Ansan-si, \\ Gyeonggi-do 15588, Republic of Korea \\ ${ }^{2}$ Molecular Phytobacteriology Laboratory, Infectious Disease Research Center, KRIBB, Daejeon 34141, Republic of Korea
}

Correspondence should be addressed to Chul Young Kim; chulykim@hanyang.ac.kr and Je-Seung Jeon; jsjeoncy@gmail.com

Received 12 July 2021; Revised 8 October 2021; Accepted 19 October 2021; Published 31 October 2021

Academic Editor: Min Li

Copyright $\odot 2021$ Ji Hoon Kim et al. This is an open access article distributed under the Creative Commons Attribution License, which permits unrestricted use, distribution, and reproduction in any medium, provided the original work is properly cited.

As important pharmaceutical resources, traditional herbal medicines retain continuous attention. To do that, isolation and identification of bioactive molecules from traditional herbal decoction are important. However, conventional fractionation through octadecyl silica column faces irreversible sample adsorption that causes a bias in bioactivity assessment. However, liquidliquid chromatographic system suffers tedious $K$ value calculation as well as insufficient capacity in separation power when crude extract composed of widely ranging polarities. Here, we developed a comprehensive linear gradient solvent system for centrifugal partition chromatography (CPC) to aid bioassay-guided isolation. The lower aqueous phase of the $n$-hexane-acetonitrile-water $(10: 2: 8, \mathrm{v} / \mathrm{v})$ was used as the stationary, whereas its upper organic phase followed by the upper phase of ethyl acetate-acetonitrilewater and water-saturated $n$-butanol-acetonitrile-water in the same ratio were eluted in a linear gradient mode, thereby increasing polarity in the mobile phase. The HPLC profiling of CPC fraction showed that proposed gradient CPC was suitable to separate metabolites from Yongdamsagan-Tang, a traditional medicinal decoction made of ten herbal plants. Exhibiting a high recovery yield of 98.3\%, antioxidant response element (ARE) luciferase-inducing assay in HepG2 cells indicated that the fractions composed of baicalein and wogonin, the marker natural products of Scutellaria baicalensis, were to be the most effective molecules from Yongdamsagan-Tang. The presented results demonstrated that bioassay-guided separation that assisted with a linear gradient CPC is an incomparable alternative to HPLC and biphasic CPC in terms of higher yield rate and redundant $K$ value calculation, respectively, which led to an unbiased/time-saving separation and identification of bioactive molecules from the complex crude extract of natural products.

\section{Introduction}

Each plant species has evolved to possess an indigenous metabolite profile. Among others, plants exhibiting pharmaceutical activities have been traditionally used as folk medicine to control various ailments. In general, a mixed herbal formulation that is composed of more than two medicinal plant species was prescribed for centuries in many Asian countries in order to elicit polyvalent pharmaceutical action or to attenuate its side-effects caused by toxic metabolites by reducing the toxicity per unit weight [1-4]. For decades, pharmacognosists have focused on the isolation and identification of the metabolites from mixed herbal formulation which led to discovering various phytochemicals. However, which metabolite or plant species among others contribute the most to the specific medicinal activity remains largely unknown.

Nowadays, contemporary interpretation of the ancient medicinal herbal formulation is important. This facilitates standardization of the ancient formulation by identifying the 
active ingredients and its composition in a mixed decoction. Thereby, pharmaceuticals can produce a quality product of natural medicine, simply by mixing active compounds that show the same medicinal property as its origins. To date, chemical fractionation conducted by resin packed column chromatography followed by bioassay screening has long been the gold standard in natural product research as a tool to simplify the complexity of the mixture and eventually to isolate the compound(s) responsible for the target bioactivities [5]. Despite its universality, innate physicochemical properties of its solid-like packing materials such as silica and octadecyl silica (ODS C18 column) continuously raised irreversible sample binding to the column that potentially lead to lose of activity or failure in (adequate) isolation of target compound(s) from crude extract $[5,6]$. Moreover, conventional bioassay-guided fractionation strategy is timeand resource-intensive.

Centrifugal partition chromatography $(\mathrm{CPC})$ can be an excellent alternative to the silica-basis column chromatography. CPC is a type of support-free liquid-liquid chromatography technique that uses an immiscible biphasic solvent system to compose stationary and mobile phase. Here, the different affinity of the target compounds for each phase (partition coefficient, $K$ value) plays a key role to fractionation. This feature allows a large sample loading capacity, no irreversible sample adsorption, and low risk in sample denaturation, thereby enhancing total sample recovery [7-11]. Our most recent study proposed a linear gradient CPC system to successfully fractionate ARE-inducing metabolites from Centipeda minima [12], a traditional medicine. In such gradient $C P C$, the $K$ values of peaks are continuously shifting due to changing polarity of mobile phase, thereby permitting separation of compounds mixture composed of widely ranging polarities, being freed from restriction of $0.5<K<2.0$ rule $[13,14]$ while performing similar to that of HPLC. More specifically, time consuming/ tedious $K$ value calculation was not needed $[12,15]$. Here, we applied this CPC solvent system to separate complex natural product mixture such as traditional Oriental decoction.

Yongdamsagan-Tang (also known as LongdanxiganTang in China and Ryutanshakan-To in Japan) is a type of mixed decoction that consists of ten herbal medicines in a certain ratio (Table 1) and is widely used for treating hepatic disease, gall bladder, congested eyes, swelling, and pain in the ear in Korea, China, and Japan [26, 27] as an Oriental medicine prescription. Previously, various plant secondary metabolites belong to widely ranging polarities such as flavonoids, phenylethanoid glycosides, iridoid glycosides, secoiridoids glycosides, coumarins, triterpenes, and triterpenoidal saponins $[28,29]$ isolated and reported from Yongdamsagan-Tang. A more recent study reported that this decoction increased antioxidant enzyme activities in prostate tissues [27]. Therefore, Yongdamsagan-Tang was chosen as a model sample to carry out comprehensive linear-gradient CPC for the identification of the antioxidant response element (ARE)-inducing compounds, followed by to identify the most contributing herbal ingredients.

In the present study, together with our recent study [12], we reported a linear gradient solvent system developed for
CPC fractionation and screened its active metabolites from a complex natural compound mixture such as Yongdamsagan-Tang as a model extract. Referring to the conventional liquid-liquid extraction, $n$-hexane, ethyl acetate, and watersaturated $n$-butanol in different ratios were chosen as less polar solvents in ternary biphasic solvent systems while acetonitrile was used as the polar modifier considering the settling time and upper/lower volume ratios of the twophase solvent system. The developed gradient solvent system demonstrated unbiased activity-guided purification with an ARE-luciferase reporter assay in HepG2 cells.

\section{Materials and Methods}

2.1. Apparatus. An SCPC-100+1000 (Armen Instrument, France) apparatus and a Spot Prep II HPLC instrument (an automated HPLC system: injector, pump, UV/Vis detector, fraction collector, and digital screen, Armen Instrument, France) were combined to form a CPC system. In this study, $1000 \mathrm{~mL}$ rotor was applied. HPLC analyses were carried out using an Agilent 1260 HPLC system (Agilent Technologies, Waldbronn, Germany) controlled by ChemStation software. It was equipped with G1322A 1260 degasser, G1312C 1260 binary pump, G1329B autosampler, G1316A column oven, and G1315D DAD detector. NMR spectra were obtained on a Bruker model digital AVANCE III 400 spectrometer (Bruker, Germany).

2.2. Reagents and Materials. All solvents used for the CPC were of analytical grade and purchased from Daejung Chemical (Gyeonggi-do, Korea). The HPLC grade solvents were obtained from Fisher Scientific (Pittsburgh, PA, USA). Ultrapure water was prepared using the Milli-Q SP water purifying system. The herbal medicines used in the experiment were purchased at Kyungdong Oriental Herbal Market (Seoul, Republic of Korea), and voucher specimens were deposited in the Herbarium of the College of Pharmacy, Hanyang University.

2.3. Yongdamsagan-Tang Extract Preparation. To prepare Yongdamsagan-Tang, a mixed decoction composed of ten herbal medicines, each herbal medicine was grinded and mixed as described in Table 1 . A total of $330 \mathrm{~g}$ of Yongdamsagan-Tang powder was extracted with $1000 \mathrm{~mL}$ of ethanol for $2 \mathrm{~h}$ at $80^{\circ} \mathrm{C}$ under reflux. Then, filtered extract was dried under a rotary evaporator and freeze dryer. The dried extract was kept at $-20^{\circ} \mathrm{C}$ before use.

\subsection{Ternary Biphasic Solvent System Preparation and Its} Physical Properties. To find a gradient elution solvent system capable of separating compounds with a wide polarity range, various solvents in different ratios were tested. In detail, common solvents used for liquid-liquid extraction ( $n$-hexane, ethyl acetate, water-saturated $n$-butanol, and water) were fixed and several polarity modifier solvents (methanol, ethanol, acetonitrile, and isopropanol) were added. The settling time, which is highly responsible for the retention of 
TABle 1: Composition of Yongdamsagan-Tang.

\begin{tabular}{|c|c|c|c|c|}
\hline Ratin name & Scientific name & $\begin{array}{l}\text { Ratio } \\
(\mathrm{g})\end{array}$ & Marker compound & Reference \\
\hline $\begin{array}{l}\text { Gentianae Scabrae } \\
\text { Radix et Rhizoma }\end{array}$ & Gentiana scabra Bunge & 30 & Gentiopicroside & {$[16]$} \\
\hline Scutellariae Radix & $\begin{array}{c}\text { Scutellaria baicalensis } \\
\text { Georgi }\end{array}$ & 40 & Baicalin, wogonoside, baicalein, wogonin & [17] \\
\hline Gardeniae Fructus & Gardenia jasminoides J.Ellis & 30 & Geniposide, gardenoside, crocetin & {$[18]$} \\
\hline Alismatis Rhizoma & $\begin{array}{c}\text { Alisma plantago-aquatica } \\
\text { subsp. Orientale (Sam.) Sam. }\end{array}$ & 30 & Alisol A, alisol A 24-acetate, alisol B 24-acetate & [19] \\
\hline Akebia Caulis & $\begin{array}{c}\text { Akebia quinata (Houtt.) } \\
\text { Decne. }\end{array}$ & 40 & $\begin{array}{l}\text { 23-Hydroxy-3 } \beta \text { - }[(O-\alpha \text {-L-rhamnopyranosyl }(1 \longrightarrow 2)-\alpha \text {-L- } \\
\text { arabinopyranosyl)oxy]olean12-en-28-oic acid } O-\alpha \text {-L- } \\
\text { rhamnopyranosyl- }(1 \longrightarrow 4) \text {-O- } \beta \text {-D-glucopyranosyl- }(1 \longrightarrow 6) \text { - } \\
\beta \text {-D-glucopyranosyl ester }\end{array}$ & {$[20]$} \\
\hline Plantaginis Semen & Plantago asiatica $\mathrm{L}$. & 40 & Plantamajoside, acetoside & {$[21]$} \\
\hline $\begin{array}{l}\text { Angelicae Gigantis } \\
\text { Radix }\end{array}$ & Angelica gigas Nakai & 30 & Decursin, decursinol angelate & {$[22]$} \\
\hline Rehmanniae Radix & $\begin{array}{l}\text { Rehmannia glutinosa } \\
\text { (Gaertn.) DC. }\end{array}$ & 50 & Aucubin, Leonuride, acteoside, echinacoside & {$[23]$} \\
\hline Bupleuri Radix & Bupleurum falcatum $\mathrm{L}$. & 30 & Saikosaponin a & {$[24]$} \\
\hline $\begin{array}{l}\text { Glycyrrhizae Radix et } \\
\text { Rhizoma }\end{array}$ & Glycyrrhiza uralensis Fisch. & 10 & Liquiritin, glycyrrhizic acid & {$[25]$} \\
\hline
\end{tabular}

the stationary phase, was expressed as the time to form a clear bilayer between the two phases $(1: 1, \mathrm{v} / \mathrm{v})$ when mixed. The phase ratio was calculated as the ratio of each phase after mixing the upper and the lower phase. The various ternary biphasic solvent systems and their physical properties are provided in Table S2.

2.5. HPLC Analysis. Yongdamsagan-Tang extract and its CPC peak fractions were analyzed by an Agilent $1260 \mathrm{HPLC}$ system with an INNO C18 column $(4.6 \times 250 \mathrm{~mm}, 5 \mu \mathrm{m}$, Young Jin Biochrom, Korea). The mobile phase consisted of acetonitrile ( $0.1 \%$ formic acid, solvent $\mathrm{A})$ and water $(0.1 \%$ formic acid, solvent $\mathrm{B}$ ) in a gradient mode: $0-50 \mathrm{~min}$, $10-100 \% \mathrm{~A} ; 60 \mathrm{~min}, 100 \% \mathrm{~A}$. The flow rate was $1 \mathrm{~mL} / \mathrm{min}$, and the injection volume was $10 \mu \mathrm{L}$. The diode array detector (DAD) measured the UV spectrum over a range of 210 to $600 \mathrm{~nm}$ whilst the chromatogram of the effluents was recorded at $254 \mathrm{~nm}$.

2.6. CPC Procedure. Preparation of the biphasic solvent system for the linear gradient elution mode was designed as described in [12]. To begin, the upper and lower phase solvents were prepared when the solvents reached a hydrostatic equilibration after mixing. The $1000 \mathrm{~mL}$ volume of the CPC rotor was filled with lower aqueous phase of $n$ hexane-acetonitrile-water $(10: 2: 8, \mathrm{v} / \mathrm{v} / \mathrm{v})$ as the stationary phase at a flow rate of $50 \mathrm{~mL} / \mathrm{min}$ in ascending mode at a rotor speed of $500 \mathrm{rpm}$. Then, the rotation speed of the rotor was accelerated to $900 \mathrm{rpm}$, and the upper organic phase as the mobile phase was carried into the rotor in descending mode at $10 \mathrm{~mL} / \mathrm{min}$. When the $\mathrm{CPC}$ rotor reached hydrostatic equilibrium, indicated by the outlet exuding a clear mobile phase $(120 \mathrm{~mL}$ of stationary phase out of $1000 \mathrm{~mL}$ rotor volume, 88 bar), the sample solution (1.25 g of YDS extract dissolved in $5 \mathrm{~mL}$ of mixed upper and lower phase) was subjected to the Armen CPC system. The CPC fractionation was carried out in gradient mode with mixed mobile phase of A (upper phase of $n$-hexane-acetonitrilewater, $10: 2: 8, \mathrm{v} / \mathrm{v} / \mathrm{v}$ ), B (upper phase of ethyl acetate-acetonitrile-water, $10: 2: 8, \mathrm{v} / \mathrm{v} / \mathrm{v}$ ), and $\mathrm{C}$ (upper phase of watersaturated $n$-butanol-acetonitrile-water, $10: 2: 8$, v/v/v) with a flow rate of $10 \mathrm{~mL} / \mathrm{min}$ : $0-50 \mathrm{~min}(100 \% \mathrm{~A}), 50-145 \mathrm{~min}$ (100\% A-100\% B), 145-250 min (100\% B-100\% C), and 250-340 min (100\% C). Thereafter, methanol for washing was pumped at $50 \mathrm{~mL} / \mathrm{min}$ to recover all the remaining samples. The linear gradient mode is depicted in Figure 1(a).

2.7. Cell Culture and Viability Assay. The construction of the HepG2-ARE cells (transfected Pgl4.37 [luc2P/ARE/Hygro] (Promega)) was carried out as previous description [12, 30]. The HepG2-ARE cells were cultured in DMEM high glucose media (Hyclone, Logan, UT) supplemented with 10\% FBS (Hyclone, Logan, UT), $1 \%$ penicillin-streptomycin (Hyclone, Logan, UT, USA) and 1\% hygromycin B (Invitrogen, Carlsbad, CA, USA). The cell viability was examined using the 3-(4,5-dimethylthiazol-2-yl)-2,5-diphenyl tetrazolium bromide (MTT) assay. HepG2 cells stably transfected with pGL4.37 (HepG2-ARE cell) were seeded at a density of $1 \times 10^{5}$ cells/well in 24 -well plates for $24 \mathrm{~h}$. After serum starvation for $12 \mathrm{~h}$ when they grew to approximately $80 \%$ confluency, the cells were treated with the major compounds and incubated for $24 \mathrm{~h}$. Thereafter, the cells were treated with $50 \mu \mathrm{L}$ of MTT for $1 \mathrm{~h}$. The formazan precipitate was dissolved in $1 \mathrm{~mL}$ of dimethyl-sulfoxide (DMSO), and the absorbance was measured at $570 \mathrm{~nm}$ using a microplate reader.

2.8. ARE-Inducing Activities Assay. HepG2-ARE cells were seeded at a density of $1 \times 10^{5}$ cells/well in 24 -well plates for $24 \mathrm{~h}$. The cells were starved for $12 \mathrm{~h}$ when they reached to approximately $80 \%$ confluency and exposed the cells to 


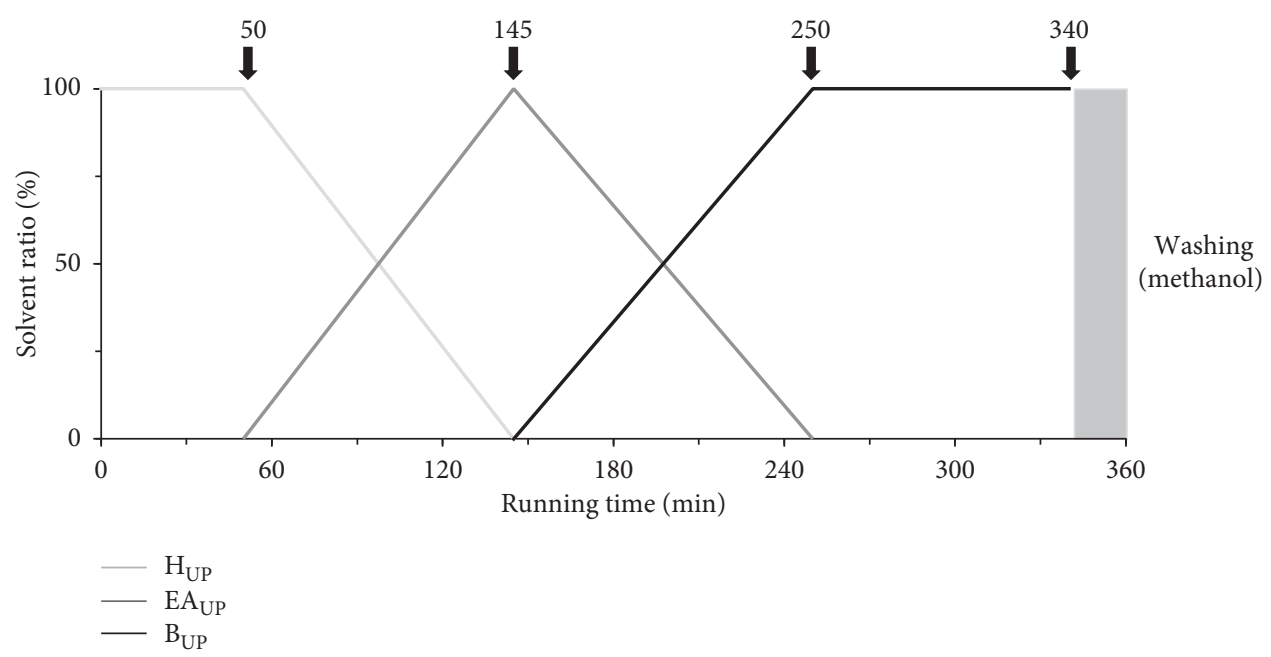

(a)

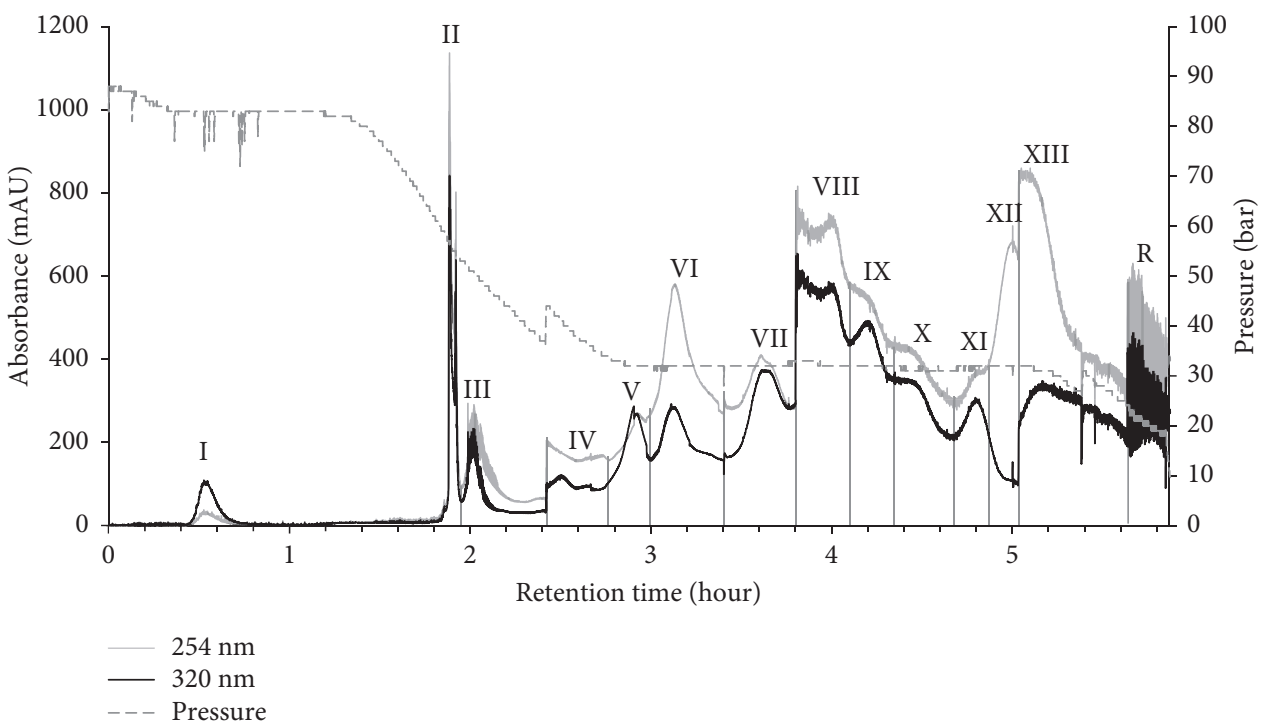

(b)

Figure 1: Gradient CPC for fractionation of Yongdamsagan-Tang (1.25 g). (a) Gradient mobile phase elution condition; (b) chromatogram of CPC separation at $254 \mathrm{~nm}$ and $320 \mathrm{~nm}$. Stationary phase: lower aqueous phase of $n$-hexane-acetonitrile-water $(10: 2: 8$, v/v/v); flow rate: $10 \mathrm{~mL} / \mathrm{min}$; rotation speed: $900 \mathrm{rpm}$; mode ascending.

crude extract, $\mathrm{CPC}$ fractions, and purified compounds for an additional $24 \mathrm{~h}$. Then, the cells were lysed with $120 \mu \mathrm{L}$ of passive lysis buffer (Promega, Madison, WI, USA) in an ice rack and transferred in $1.5 \mathrm{~mL}$ tubes. The tubes were then centrifuged at $1000 \mathrm{rpm}$ for $3 \mathrm{~min}$. Each supernatant $(30 \mu \mathrm{L})$ in the centrifuged tube was reacted with $60 \mu \mathrm{L}$ of luciferase assay substrate (Promega, Madison, WI, USA) in a white 96well plate. Finally, luminescence was measured by an EnSpire multimode plate reader (PerkinElmer, Waltham, MA, USA). DMSO (below $0.1 \%$ ) was used as a vehicle, which was the negative control. Sulforaphane $(5 \mu \mathrm{M})$ (Calbiochem, Darmstadt, Germany) was used as the positive control.

2.9. Protein Assay. The protein was determined by the Pierce Micro BCA Protein Assay Kit with BSA as a standard (Pierce No. 23227; Thermo Fisher Scientific, Illinois, USA). Each standard and unknown sample lysate $(10 \mu \mathrm{L})$ was replicated into a 96-well microplate. The working reagent $(200 \mu \mathrm{L})$ was added to each well. BCA reagents $\mathrm{A}$ and $\mathrm{B}$ were mixed in a 49-to-1 volume ratio. After $30 \mathrm{~min}$ of incubation at $37^{\circ} \mathrm{C}$, the plate was cooled at room temperature and the absorbance was measured at $562 \mathrm{~nm}$ on a microplate reader. The calculated value of the protein assay was used as a factor to normalize the ARE-inducing activity.

2.10. Statistical Analysis. All data are reported as mean$s \pm$ S.E. and the statistical significance of the differences between the treatments was assessed using the student's $t$ test. ${ }^{*} p<0.05,{ }^{* *} p<0.01$, and ${ }^{* * *} p<0.005$ were considered statistically significant. The relative luciferase activity of aglycones (baicalein and wogonin) and their glycosides (baicalin and wogonoside) to $5 \mu \mathrm{M}$ sulforaphane (\%) in 
different combinations were analyzed with $\mathrm{R}$ Studio software (Version 4.1.1). To meet normality and homogeneity of variance, the data were transformed using Box-Cox using a package MASS. Differences were tested by two-way analysis of variance (ANOVA). A Tukey-HSD test was used to separate group mean values when the ANOVA was significant at $p<0.05$ (triplicates).

\section{Results and Discussion}

3.1. Gradient Biphasic Solvent Systems Selection. In CPC separation, selection of an appropriate biphasic solvent system is key to success. As traditional Oriental herbal decoction such as Yongdamsagan-Tang contains a myriad of phytochemicals covering diverse polarities, solvent system satisfying isolation of compounds with polymorphous polarities is needed. To do that, a serious of ternary biphasic solvent system was tested to develop a comprehensive linear gradient solvent system for CPC. Considering the water is common for all the solvent systems, the aqueous phase was used as a stationary phase while the organic phase was used for mobile phase with increased polarities. The magnitude of polarity of organic phase was gradually enhanced with $n$ hexane, ethyl acetate, and $n$-butanol. As polarity modifier, methanol, ethanol, acetonitrile, and isopropanol were tested. The results showed that among others, acetonitrile exhibited to be a best polarity modifier in terms of the least settling time compared to others, as described in Table S2. On the other hand, isopropanol as a polar modifier was not suitable for CPC experiment due to high initial pressure, deterring mobile phase outflow, altogether gradient ternary biphasic solvent systems composed of $n$-hexane-acetonitrile-water $(10: 2: 8, \mathrm{v} / \mathrm{v} / \mathrm{v})$, ethyl acetate-acetonitrile-water $(10: 2: 8, \mathrm{v} / \mathrm{v} /$ $\mathrm{v})$, and water-saturated $n$-butanol-acetonitrile-water $(10: 2$ : 8 ) were chosen as apolar, semipolar, and polar conditions, respectively.

\subsection{Separation of the CPC Gradient Elution and ARE-Inducing} Activity. In order to unearth ARE-inducing compounds from Yongdamsagan-Tang, we preliminary evaluated AREluciferase activity of each herbal medicine. Among others, Scutellaria baicalensis, Angelica gigas, and Glycyrrhiza uralensis exhibited to induce ARE activities (Figure 2(c)). It was shown that $S$. baicalensis, A. gigas, and G. uralensis induced an ARE via nuclear factor erythroid 2-related factor 2 (Nrf2) [31]. Here, baicalein, baicalin, and oroxylin A in $S$. baicalensis were reported to be the inducers of the Nrf2/ ARE pathway, while in A. gigas, decursin and decursinol angelate exerted neuroprotective effects against oxidative stress which is induced by amyloid $\beta$-protein in PC12 cells via Nrf2-mediated upregulation of heme oxygenase-1 [32]. In $G$. uralensis, isoliquiritigenin was reported as the most potent ARE inducer [33]. However, it is difficult to reveal the active components in Yongdamsagan-Tang, since Yongdamsagan-Tang is a mixture of ten herbal medicines, which are mixtures of thousands of phytochemicals with a wide range of polarities. Therefore, we applied recently proposed comprehensive gradient CPC method [12] to search AREinducing compounds from Yongdamsagan-Tang.

CPC separation was carried out in a linear gradient elution of three ternary solvent systems (Figure 1(a)). For the mobile phase, the upper organic phases from $n$-hexaneacetonitrile-water $(10: 2: 8, \mathrm{v} / \mathrm{v})$, ethyl acetate-acetonitrilewater $(10: 2: 8, \mathrm{v} / \mathrm{v})$, and water-saturated $n$-butanol-acetonitrile-water $(10: 2: 8)$ were sequentially introduced in ascending mode. As shown in Figure 1(b), fourteen fractions (I-XIII and R) were obtained at high resolution for $360 \mathrm{~min}$ operating time. The initial operating pressure was about 90 bar, then it began to decline after 80 min until $150 \mathrm{~min}$, and it was then maintained at 32 bar for approximately $180 \mathrm{~min}$. According to the CPC chromatogram, fourteen fractions were collected and concentrated to obtain the fractions I (27.5-45 min, $45.6 \mathrm{mg})$, II (110-117.5 min, $90.2 \mathrm{mg}$ ), III (117.5-145 min, $54.7 \mathrm{mg})$, IV (145-160 min, $10.3 \mathrm{mg}), \quad$ V (160-180 min, $11.0 \mathrm{mg})$, VI (180-205 min, $120.7 \mathrm{mg}), \quad$ VII $\quad(205-222.5 \mathrm{~min}, \quad 21.7 \mathrm{mg}), \quad$ VIII (222.5-230 min, $32.0 \mathrm{mg}$ ), IX (230-260 min, $22.1 \mathrm{mg}), \mathbf{X}$ (260-280 min, $18.0 \mathrm{mg})$, XI (280-295 min, $17.1 \mathrm{mg})$, XII (295-302.5 min, $34.9 \mathrm{mg}$ ), XIII (302.5-340 min, $25.3 \mathrm{mg}$ ), and $\mathbf{R}$ (residue in rotor, $725.5 \mathrm{mg}$ ). Collectively, the total sum of all the CPC fractionations was 1,129.1 mg out of 1,250 mg crude extract, exhibiting a high recovery yield of $98.3 \%$.

Figure 3 displays the HPLC chromatogram of each CPC peak fraction from I to $\mathbf{R}$ at $254 \mathrm{~nm}$. Fractions I, II, III, VI, VII, XII, and XIII were well corresponded to include the representative Yongdamsagan-Tang peaks 1-7 in reverse order. In addition, several fractions presented to be pure with a high purity such as fraction II (wogonin $6,>86 \%$ ); fraction III (baicalein 5, 86\%); fraction VII (baicalin 3, 88\%); and fraction XII (geniposide 1, 89\%). To sort out active fractions, the crude sample of Yongdamsagan-Tang and its 14 CPC fractions (I-XIII and $\mathbf{R}$ fractions) were subjected to ARE-inducing activity assay at a concentration of $30 \mu \mathrm{g} / \mathrm{mL}$. To begin with, fraction III exerted the highest activity ( $>50$ folds increase) which is followed by fractions II, I, and VII (Figure 3). Interestingly, the major compounds from peak fractions II, III, and VII were from S. baicalensis, the best performing herbal medicine (in Figure 2(c)), while components from I were rooted in A. gigas, the second most effective herbal medicine to ARE-inducing activity. We then further purified the active fractions I-III and VII by preparative HPLC to obtain pure decursin (7), wogonin (6), baicalein (5), and baicalin (3), respectively. On the other hand, inactive but marker compounds geniposide (1), getiopicroside (2), and wogonoside (4) from Yongdamsagan-Tang were also purified to compare further ARE-inducing activity from the compound level. The physicochemical information of these compounds is indicated in the section Structural Information in Supplementary Materials.

The ability of seven purified Yongdamsagan-Tang compounds to enhance ARE activity was evaluated by luciferase assay in the HepG2 cells at different concentrations. No cytotoxic activity was found (Figure 4(a)). As shown in Figures 4(b) and 4(c), baicalein (5) and wogonin (6) significantly enhanced ARE activity in a dose-dependent 

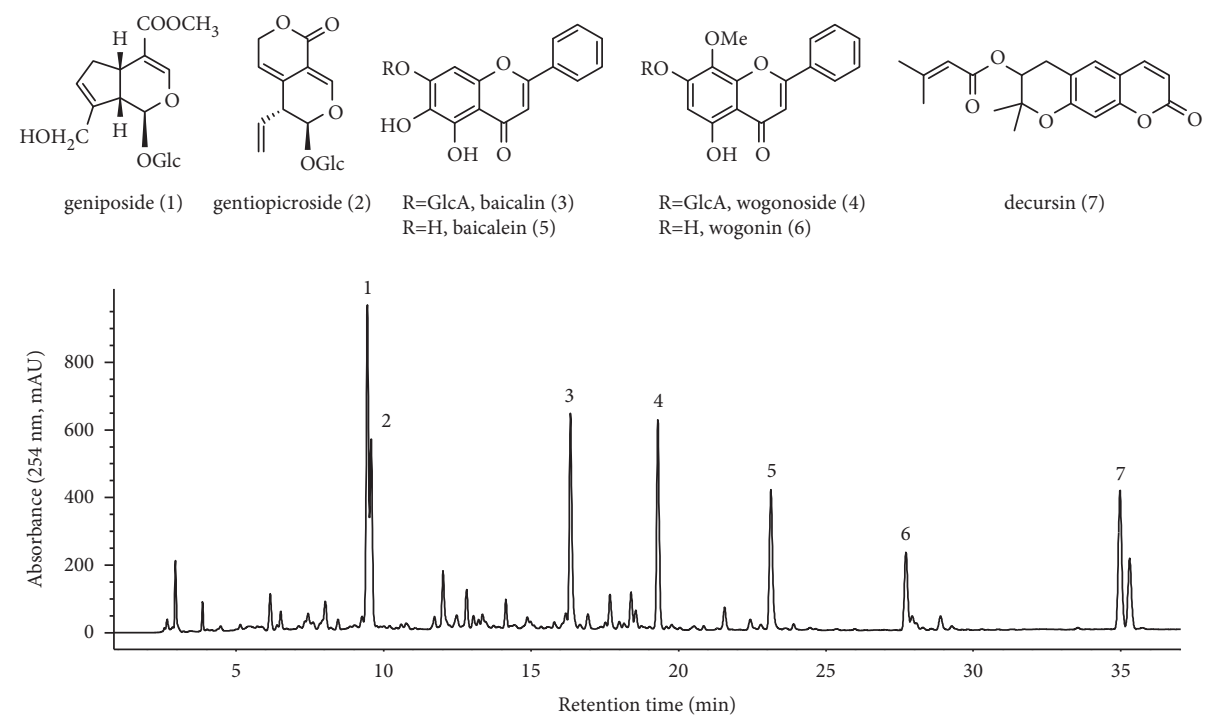

(a)

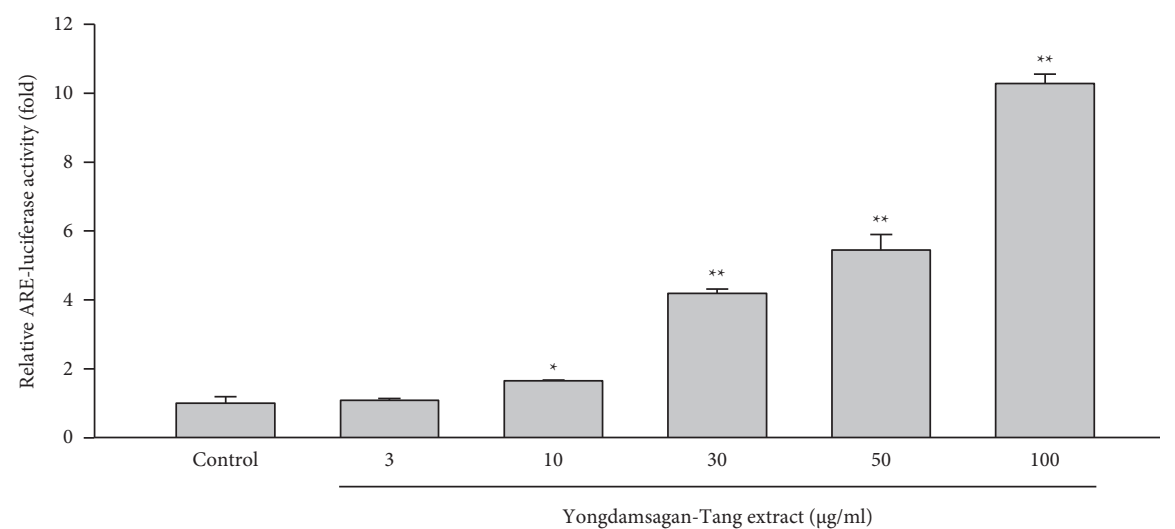

(b)

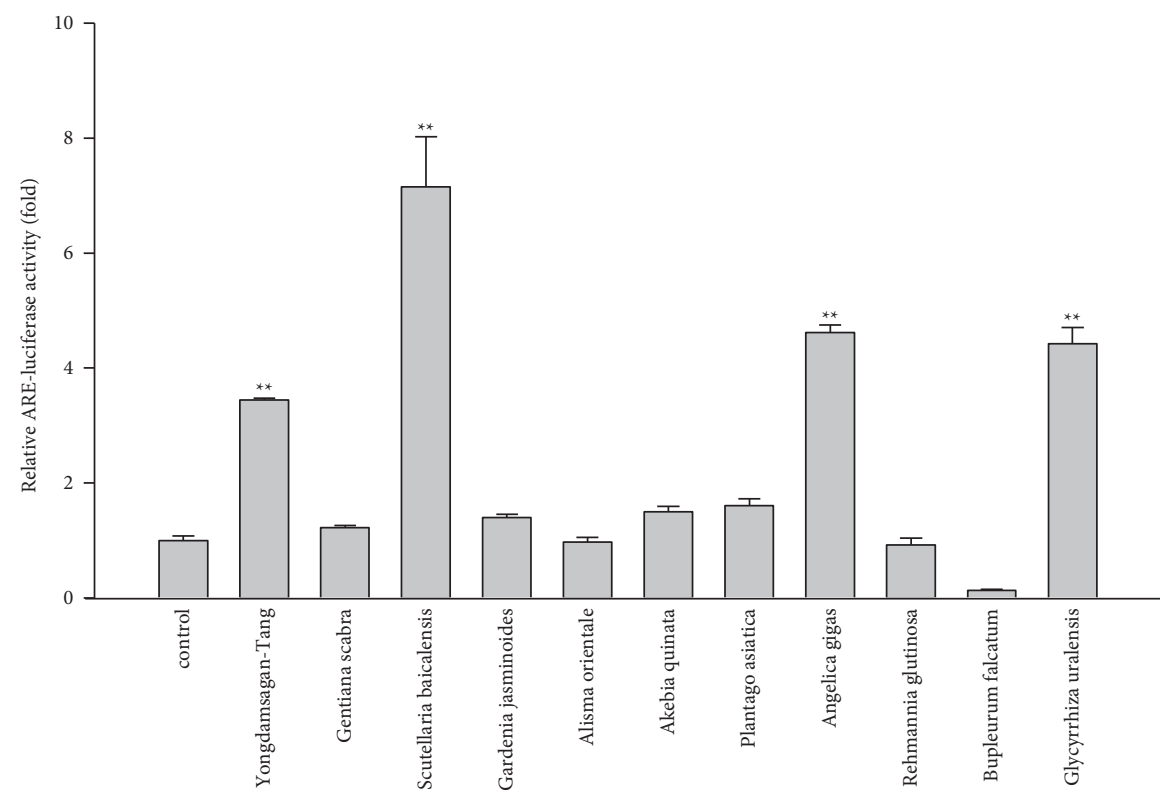

(c)

FIgURE 2: HPLC profile and relative ARE-luciferase activity of Yongdamsagan-Tang. (a) HPLC chromatogram and chemical structures of marker compounds (1-7) of Yongdamsagan-Tang; (b) relative ARE-luciferase activity of Yongdamsagan-Tang; (c) relative ARE-luciferase activity of the ingredient herbal medicines of Yongdamsagan-Tang. Data are presented as means \pm S.E. $(n=3)$. ${ }^{* *} p<0.01$ (compared with the vehicle-treated control). 


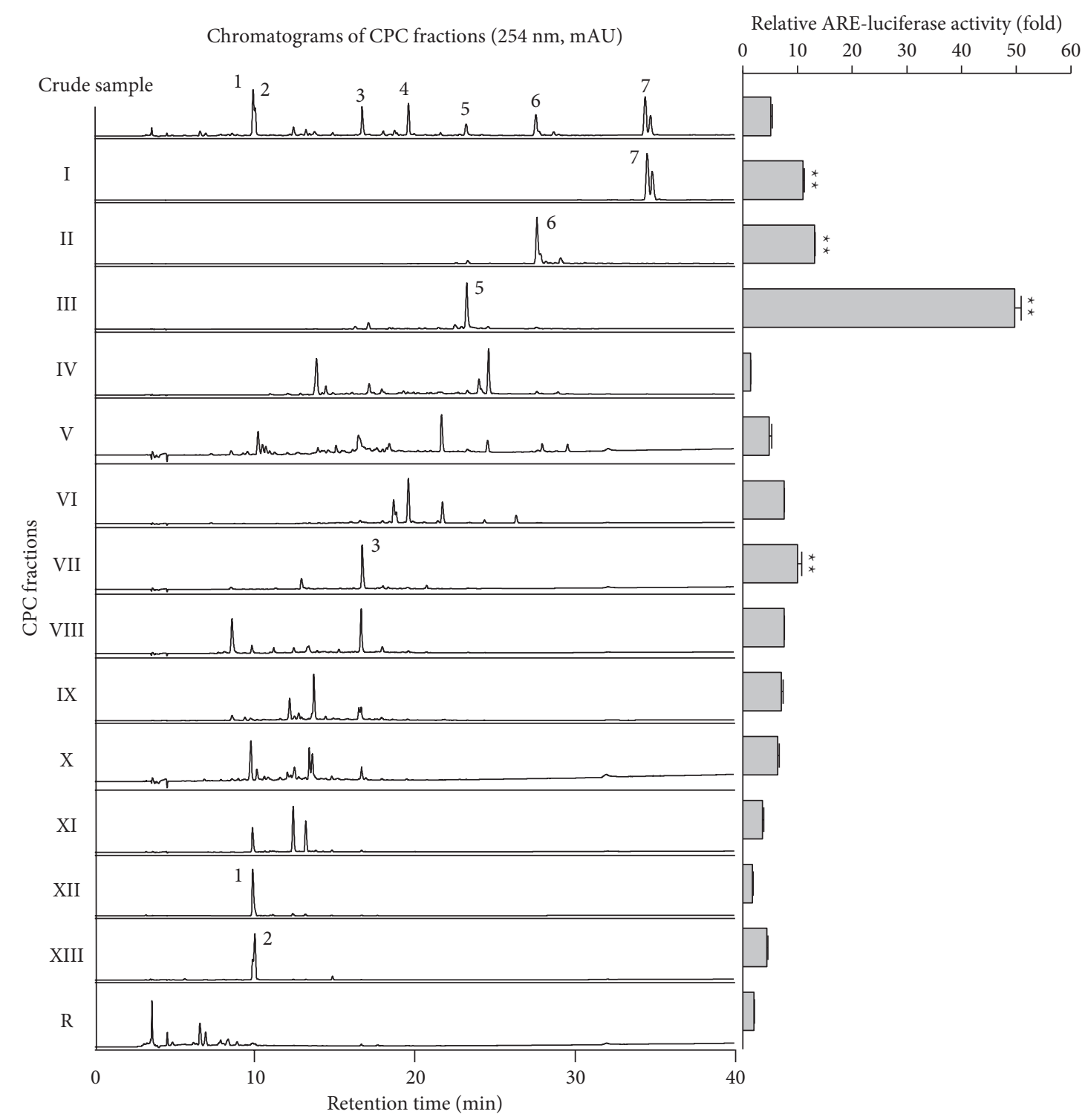

Figure 3: HPLC chromatograms and relative ARE-luciferase activity of CPC fractions at $30 \mu \mathrm{g} / \mathrm{mL}$ concentrations. Each CPC fraction was analyzed by HPLC, and its ARE-inducing activity was evaluated in ARE-HepG2 cells. Data are presented as means \pm S.E. $(n=3)$. ${ }^{* *} p<0.01$ (compared with the vehicle-treated control).

manner. At $50 \mu \mathrm{M}$, baicalein (5) enhanced ARE activity 28.9 fold (sulforaphane $5 \mu \mathrm{M}, 30.0$ fold), while wogonin (6) induced 24.0 fold (sulforaphane $5 \mu \mathrm{M}, 18.1$ fold). In addition, baicalin (3) and wogonoside (4), glycosides of baicalein and wogonin, respectively, also exhibited induced ARE activity, but their potencies were less than those of aglycones (Figure 4(c)). Geniposide (1) and decursin (7) also presented ARE-inducing activity. Overall, the results showed that baicalein (5) and wogonin (6) from S. baicalensis were major contributor to ARE-inducing activity of YongdamsaganTang and this was in line with ARE-luciferase activity of each medicinal plant, as shown in Figure 2(c). Other medicinal plant species A. gigas showed a moderate contribution to ARE activity of Yongdamsagan-Tang while G. uralensis was deemed to have least or no contribution to this decoction.
Our CPC-aided activity-guided fractionation clearly indicated that baicalein (5) and wogonin (6) are major components responsible for enhancing ARE activity of Yongdamsagan-Tang, a traditional decoction of ten traditional medicines. Here, together with our previous study [12], a linear gradient CPC system was extensively applied to fractionate a complex compounds pool composed of diverse polarities such as mixed herbal decoctions. Without irreversible sample adsorption or loss, this separation system provided the higher recovery rate which in turn contributed to unbiased activity-guided fractionation. Thus, our study highlights that linear gradient CPC system is an effective alternative to preparative HPLC in activity-guided fraction practice and this platform can be associated to other bioactivity assays as a reliable means of unbiased fractionation tool. 


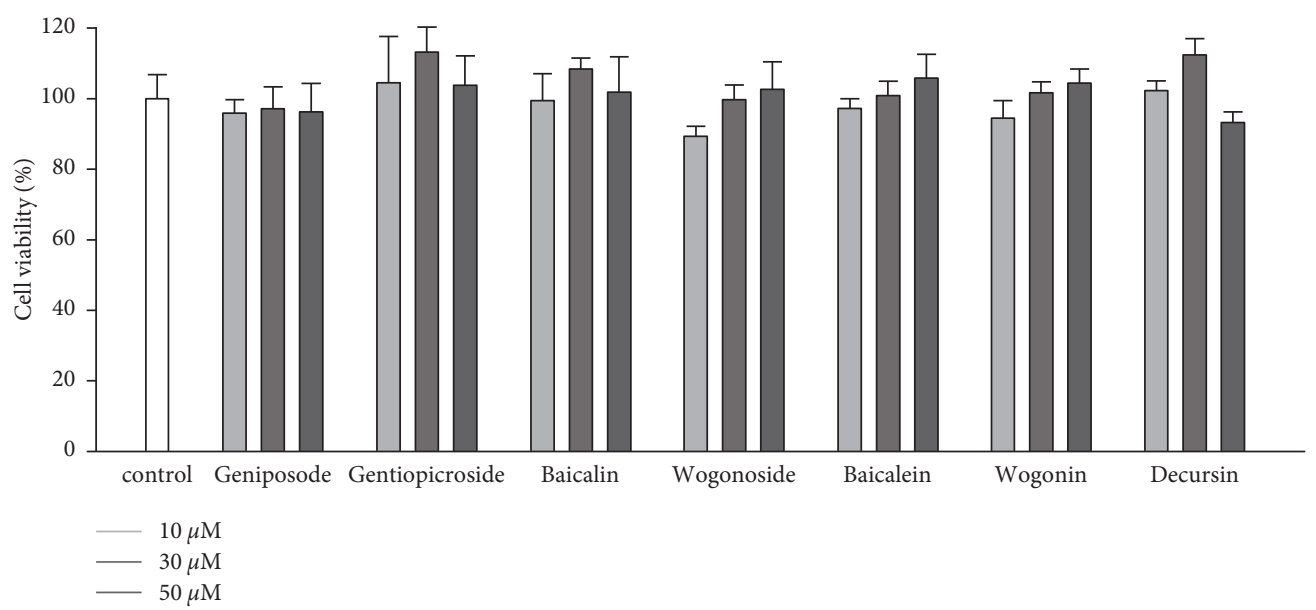

(a)
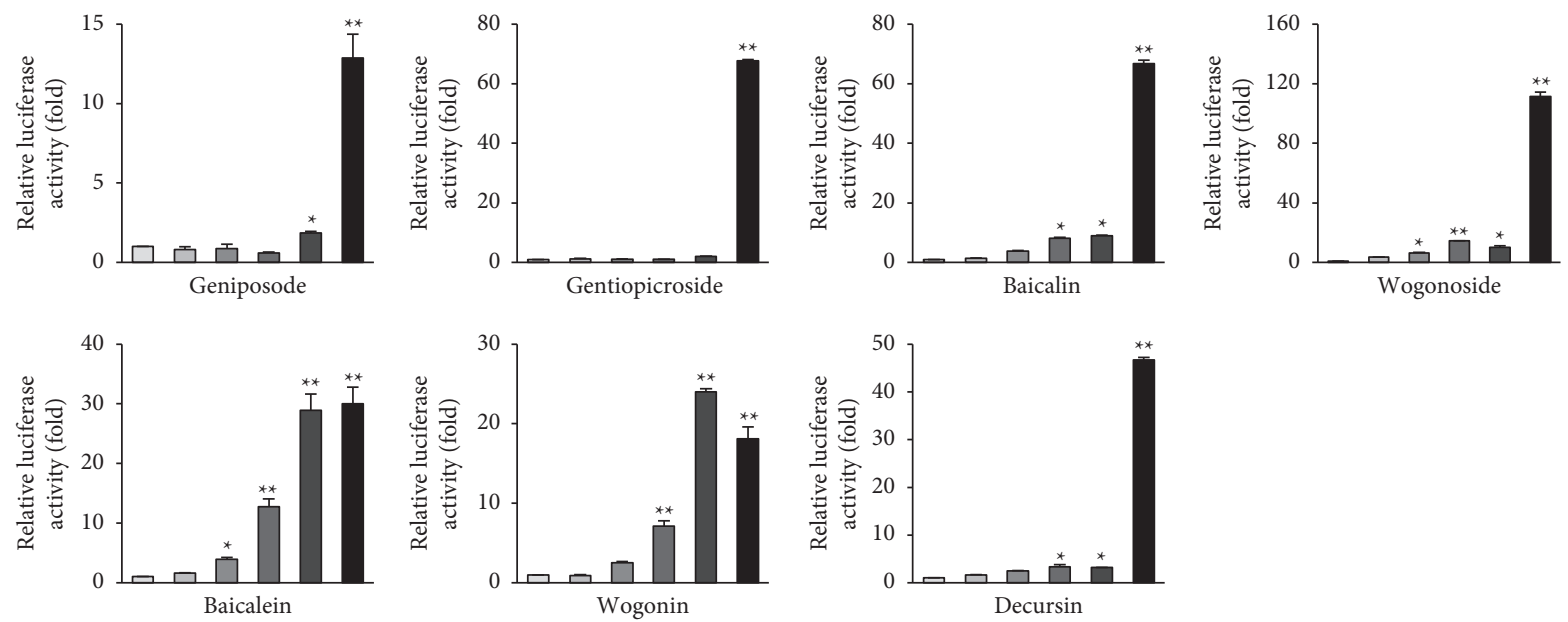

sulforaphane

$\begin{array}{cc}\text { control } & -30 \mu \mathrm{M} \\ -1 \mu \mathrm{M} & -50 \mu \mathrm{M} \\ -10 \mu \mathrm{M} & -5 \mu \mathrm{M}\end{array}$

(b)

Figure 4: Continued. 


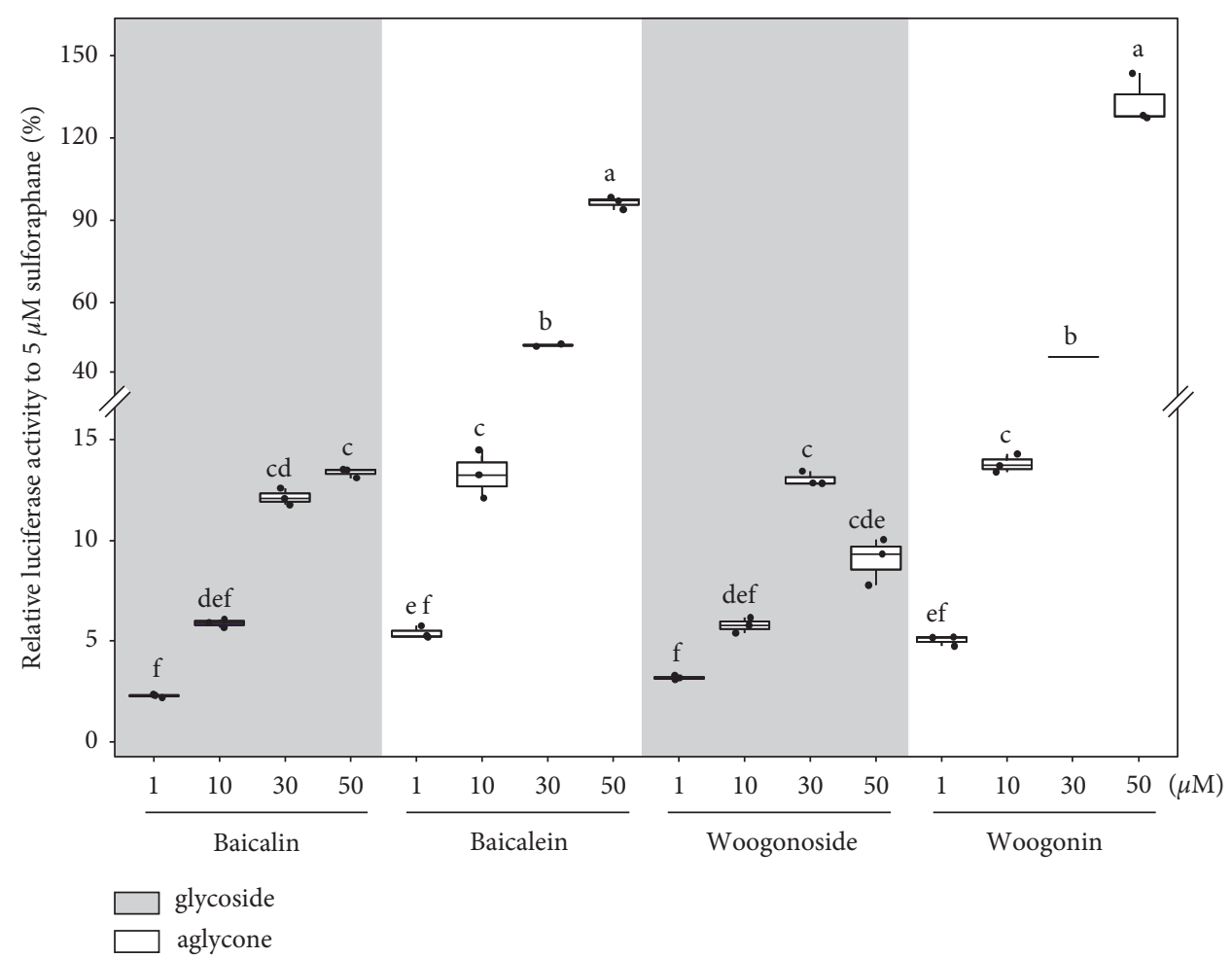

(c)

FIGURE 4: Effects of purified compounds (1-7) on ARE-luciferase activity in HepG2 cells. (a) Cytotoxicity of compounds 1-7; (b) the relative ARE-luciferase activity of isolated compounds. Data are presented as means \pm S.E $(n=3)$. (c) The relative luciferase activity of baicalein and wogonin and their glycosides baicalin and wogonoside to $5 \mu \mathrm{M}$ sulforaphane $(\%)$. ${ }^{*} p<0.05$ and ${ }^{* *} p<0.01$, (compared with the vehicletreated control). Different letters show significant difference between the treatments (two-way ANOVA, Tukey's HSD post hoc test, $p<0.05$, mean \pm standard error, $n=3$ ).

\section{Conclusions}

In this study, a comprehensive gradient solvent system for CPC was developed to aid activity-guided fractionation of Yongdamsagan-Tang, a mixture of ten herbal medicines. It was shown that a gradient elution of the upper phases of three ternary solvent system with $n$-hexane-acetonitrilewater, ethyl acetate-acetonitrile-water, and water-saturated $n$-butanol-acetonitrile-water (each $10: 2: 8, \mathrm{v} / \mathrm{v} / \mathrm{v}$ ) enabled $\mathrm{CPC}$ to fractionate Yongdamsagan-Tang, and among others, baicalein (5) and wogonin (6) were the most prominent ARE inducer in our assay system. Considering that CPC has no irreversible sample adsorption, this platform can be an effective method that aids unbiased screening of bioactive natural products from complex metabolite mixtures such as traditional Oriental decoction.

\section{Data Availability}

The data used to support the findings of this study are included within the article.

\section{Conflicts of Interest}

No potential conflicts of interest were disclosed by the authors.

\section{Authors' Contributions}

J.H.K. and C.Y.K. conceptualized the study; J.H.K. and J.-S.J. curated the data; J.H.K., E.J.J., and Y.J.L. performed formal analysis; C.Y.K. was responsible for funding acquisition; J.H.K. and E.J.J. investigated the study; J.H.K. developed the methodology; C.Y.K. did project administration; E.J.J. provided resources; J.H.K. and J.-S.J. were responsible for software; C.Y.K. supervised the study; C.Y.K. validated the study; J.H.K. visualized the study; J.H.K. wrote the original draft; J.-S.J. and C.Y.K. reviewed and edited the original draft. All authors have read and agreed to the published version of the manuscript.

\section{Acknowledgments}

This research was supported by the Basic Science Research Program through the National Research Foundation of Korea (NRF-2020R1A2C1009455 and NRF2020R1A6A1A03042854).

\section{Supplementary Materials}

Figure S1. HPLC chromatograms of Yongdamsagan-Tang and its individual herbal components. Table S1. The partition coefficients ( $\mathrm{K}$ values) of major compounds in 
Yongdamsagan-Tang. Table S2. Phase ratios and settling times of each ternary biphasic solvent system tested for a linear gradient solvent system. Physicochemical/structural information of purified metabolites from YongdamsaganTang. (Supplementary Materials)

\section{References}

[1] Q. Gao, J. Li, J. K. H. Cheung et al., "Verification of the formulation and efficacy of Danggui buxue tang (a decoction of Radix Astragali and Radix Angelicae Sinensis): an exemplifying systematic approach to revealing the complexity of Chinese herbal medicine formulae," Chinese Medicine, vol. 2, pp. 1-10, 2007.

[2] L. Luo, J. Nong Wang, L. D. Kong, Q. G. Jiang, and R. X. Tan, "Antidepressant effects of Banxia Houpu decoction, a traditional Chinese medicinal empirical formula," Journal of Ethnopharmacology, vol. 73, no. 1-2, pp. 277-281, 2000.

[3] M. I. Thabrew, R. R. Mitry, M. A. Morsy, and R. D. Hughes, "Cytotoxic effects of a decoction of Nigella sativa, Hemidesmus indicus and Smilax glabra on human hepatoma HepG2 cells," Life Sciences, vol. 77, no. 12, pp. 1319-1330, 2005.

[4] L. Yang, Y. Wang, L. Wang, H. Xiao, Z. Wang, and Z. Hu, "Rapid quantification of iridoid glycosides analogues in the formulated Chinese medicine Longdan Xiegan Decoction using high-performance liquid chromatography coupled with mass spectromentry," Journal of Chromatography A, vol. 1216, no. 11, pp. 2098-2103, 2009.

[5] J. J. Kellogg, D. A. Todd, J. M. Egan et al., "Biochemometrics for natural products research: comparison of data analysis approaches and application to identification of bioactive compounds," Journal of Natural Products, vol. 79, no. 2, pp. 376-386, 2016.

[6] L.-F. Nothias, M. Nothias-Esposito, R. da Silva et al., "Bioactivity-based molecular networking for the discovery of drug leads in natural product bioassay-guided fractionation," Journal of Natural Products, vol. 81, no. 4, pp. 758-767, 2018.

[7] Q.-B. Han, Y. Zhou, C. Feng et al., "Bioassay guided discovery of apoptosis inducers from gamboge by high-speed countercurrent chromatography and high-pressure liquid chromatography/electrospray ionization quadrupole time-of-flight mass spectrometry," Journal of Chromatography B, vol. 877, no. 4, pp. 401-407, 2009.

[8] S. Ignatova, N. Sumner, N. Colclough, and I. Sutherland, "Gradient elution in counter-current chromatography: a new layout for an old path," Journal of Chromatography A, vol. 1218, no. 36, pp. 6053-6060, 2011.

[9] K. A. Alvi, "Screening natural products: bioassay-directed isolation of active components by dual-mode ccc," Journal of Liquid Chromatography \& Related Technologies, vol. 24, no. 11-12, pp. 1765-1773, 2001.

[10] Y.-Q. Zhang, J.-G. Luo, C. Han, J.-F. Xu, and L.-Y. Kong, "Bioassay-guided preparative separation of angiotensinconverting enzyme inhibitory C-flavone glycosides from Desmodium styracifolium by recycling complexation highspeed counter-current chromatography," Journal of Pharmaceutical and Biomedical Analysis, vol. 102, pp. 276-281, 2015.

[11] A. Abedini, S. Chollet, A. Angelis et al., "Bioactivity-guided identification of antimicrobial metabolites in Alnus glutinosa bark and optimization of oregonin purification by centrifugal partition chromatography," Journal of Chromatography B, vol. 1029-1030, pp. 121-127, 2016.
[12] J. H. Kim, E. J. Jung, Y. J. Lee, E. M. Gao, A. S. Syed, and C. Y. Kim, "Bioassay-guided separation of Centipeda minima using comprehensive linear gradient centrifugal partition chromatography," Molecules, vol. 25, no. 13, p. 3077, 2020.

[13] Y. Zhang, C. Liu, Z. Zhang, J. Wang, G. Wu, and S. Li, "Comprehensive separation and identification of chemical constituents from Apocynum venetum leaves by high-performance counter-current chromatography and high performance liquid chromatography coupled with mass spectrometry," Journal of Chromatography B, vol. 878, no. 30, pp. 3149-3155, 2010.

[14] H. Ying, H. Jiang, H. Liu, F. Chen, and Q. Du, "Ethyl acetaten-butanol gradient solvent system for high-speed countercurrent chromatography to screen bioactive substances in okra," Journal of Chromatography A, vol. 1359, pp. 117-123, 2014.

[15] Z. Wang, S. H. Hwang, and S. S. Lim, "Comprehensive profiling of minor tyrosinase inhibitors from Gastrodia elata using an off-line hyphenation of ultrafiltration, high-speed countercurrent chromatography, and high-performance liquid chromatography," Journal of Chromatography A, vol. 1529, pp. 63-71, 2017.

[16] R.-W. Jiang, K.-L. Wong, Y.-M. Chan, H.-X. Xu, P. P.-H. But, and P.-C. Shaw, "Isolation of iridoid and secoiridoid glycosides and comparative study on Radix gentianae and related adulterants by HPLC analysis," Phytochemistry, vol. 66, no. 22, pp. 2674-2680, 2005.

[17] H. Bochorááková, H. Paulová, J. Slanina, P. Musil, and E. Táborská, "Main flavonoids in the root of Scutellaria baicalensis cultivated in Europe and their comparative antiradical properties," Phytotherapy Research, vol. 17, pp. 640644, 2003.

[18] M. C. Bergonzi, C. Righeschi, B. Isacchi, and A. R. Bilia, "Identification and quantification of constituents of gardenia jasminoides ellis (zhizi) by HPLC-DAD-ESI-MS," Food Chemistry, vol. 134, no. 2, pp. 1199-1204, 2012.

[19] X. Liu, S.-L. Li, Y. Zhou et al., "Characterization of protostane triterpenoids inAlisma orientalisby ultra-performance liquid chromatography coupled with quadrupole time-of-flight mass spectrometry," Rapid Communications in Mass Spectrometry, vol. 24, no. 11, pp. 1514-1522, 2010.

[20] Y. Ling, Y. Zhang, Y. Zhou, D. Jiang, L. Xu, and L. Liao, "Rapid detection and characterization of the major chemical constituents in Akebia quinata by high performance liquid chromatography coupled to electrospray ionization and quadrupole time-of-flight mass spectrometry," Analytical Methods, vol. 8, no. 12, pp. 2634-2644, 2016.

[21] L. Li, C. Liu, Z. Liu, R. Tsao, and S. Liu, "Identification of phenylethanoid glycosides in plant extract ofPlantago asiaticaL. By liquid chromatography-electrospray ionization mass spectrometry," Chinese Journal of Chemistry, vol. 27, no. 3, pp. 541-545, 2009.

[22] M.-J. Ahn, M. K. Lee, Y. C. Kim, and S. H. Sung, "The simultaneous determination of coumarins in Angelica gigas root by high performance liquid chromatography-diode array detector coupled with electrospray ionization/mass spectrometry," Journal of Pharmaceutical and Biomedical Analysis, vol. 46, no. 2, pp. 258-266, 2008.

[23] S. Tong, L. Chen, Q. Zhang, J. Liu, J. Yan, and Y. Ito, "Separation of catalpol from rehmannia glutinosa libosch. By high-speed countercurrent chromatography," Journal of Chromatographic Science, vol. 53, no. 5, pp. 725-729, 2015.

[24] K. D. Yoon and J. Kim, "Application of centrifugal partition chromatography coupled with evaporative light scattering 
detection for the isolation of saikosaponins-a and -c fromBupleurum falcatumroots," Journal of Separation Science, vol. 32, no. 1, pp. 74-78, 2009.

[25] W. C. Liao, Y.-H. Lin, T.-M. Chang, and W.-Y. Huang, "Identification of two licorice species, Glycyrrhiza uralensis and Glycyrrhiza glabra, based on separation and identification of their bioactive components," Food Chemistry, vol. 132, no. 4, pp. 2188-2193, 2012.

[26] E. Park, M.-Y. Lee, C.-S. Seo, W.-Y. Jeon, and H.-K. Shin, "Yongdamsagan-tang, a traditional herbal formula, inhibits cell growth through the suppression of proliferation and inflammation in benign prostatic hyperplasia epithelial-1 cells," Journal of Ethnopharmacology, vol. 209, pp. 230-235, 2017.

[27] E. Park, M.-Y. Lee, W.-Y. Jeon, N. Lee, C.-S. Seo, and H.-K. Shin, "Inhibitory effect of yongdamsagan-tang water extract, a traditional herbal formula, on testosterone-induced benign prostatic hyperplasia in rats," Evidence-Based Complementary and Alternative Medicine, vol. 2016, Article ID 1428923, 8 pages, 2016.

[28] Y. Wang, L. Kong, L. Hu et al., "Biological fingerprinting analysis of the traditional Chinese prescription Longdan Xiegan Decoction by on/off-line comprehensive two-dimensional biochromatography," Journal of Chromatography $B$, vol. 860, no. 2, pp. 185-194, 2007.

[29] C.-S. Seo and H.-K. Shin, "Simultaneous quantification of eight marker compounds in yongdamsagan-tang using a high-performance liquid chromatography equipped with photodiode array detector," Journal of Chromatographic Science, vol. 55, no. 9, pp. 926-933, 2017.

[30] J.-S. Jeon, C. L. Park, A. S. Syed, Y.-M. Kim, I. J. Cho, and C. Y. Kim, "Preparative separation of sesamin and sesamolin from defatted sesame meal via centrifugal partition chromatography with consecutive sample injection," Journal of Chromatography B, vol. 1011, pp. 108-113, 2016.

[31] H. Kumar, I.-S. Kim, S. V. More, B.-W. Kim, and D.-K. Choi, "Natural product-derived pharmacological modulators of Nrf2/ARE pathway for chronic diseases," Natural Product Reports, vol. 31, no. 1, pp. 109-139, 2014.

[32] L. Li, W. Li, S.-W. Jung, Y.-W. Lee, and Y.-H. Kim, "Protective effects of decursin and decursinol angelate against amyloid $\beta$-protein-induced oxidative stress in the PC12 cell line: the role of Nrf2 and antioxidant enzymes," Bioscience, Biotechnology, and Biochemistry, vol. 75, no. 3, pp. 434-442, 2011.

[33] H. Gong, B.-K. Zhang, M. Yan et al., "A protective mechanism of licorice (Glycyrrhiza uralensis): isoliquiritigenin stimulates detoxification system via Nrf2 activation," Journal of Ethnopharmacology, vol. 162, pp. 134-139, 2015. 